\title{
Local semicircle law and complete delocalization for Wigner random matrices
}

\author{
László Erdős ${ }^{1}$, Benjamin Schlein ${ }^{1 *}$ and Horng-Tzer Yau ${ }^{2 \dagger}$ \\ Institute of Mathematics, University of Munich, \\ Theresienstr. 39, D-80333 Munich, Germany ${ }^{1}$ \\ Department of Mathematics, Harvard University \\ Cambridge MA 02138, USA $^{2}$
}

Jun 16, 2008

\begin{abstract}
We consider $N \times N$ Hermitian random matrices with independent identical distributed entries. The matrix is normalized so that the average spacing between consecutive eigenvalues is of order $1 / N$. Under suitable assumptions on the distribution of the single matrix element, we prove that, away from the spectral edges, the density of eigenvalues concentrates around the Wigner semicircle law on energy scales $\eta \gg N^{-1}(\log N)^{8}$. Up to the logarithmic factor, this is the smallest energy scale for which the semicircle law may be valid. We also prove that for all eigenvalues away from the spectral edges, the $\ell^{\infty}$-norm of the corresponding eigenvectors is of order $O\left(N^{-1 / 2}\right)$, modulo logarithmic corrections. The upper bound $O\left(N^{-1 / 2}\right)$ implies that every eigenvector is completely delocalized, i.e., the maximum size of the components of the eigenvector is of the same order as their average size.

In the Appendix, we include a lemma by J. Bourgain which removes one of our assumptions on the distribution of the matrix elements.
\end{abstract}

AMS Subject Classification: 15A52, 82B44

Running title: Local semicircle law

Key words: Semicircle law, Wigner random matrix, random Schrödinger operator, density of states, localization, extended states.

\footnotetext{
*Supported by Sofja-Kovalevskaya Award of the Humboldt Foundation. On leave from Cambridge University, UK

${ }^{\dagger}$ Partially supported by NSF grant DMS-0602038
} 


\section{Introduction}

The Wigner semicircle law states that the empirical density of the eigenvalues of a random matrix is given by the universal semicircle distribution. This statement has been proved for many different ensembles, in particular for the case when the distributions of the entries of the matrix are independent, identically distributed (i.i.d.). To fix the scaling, we normalize the matrix so that the bulk of the spectrum lies in the energy interval $[-2,2]$, i.e., the average spacing between consecutive eigenvalues is of order $1 / N$. We now consider a window of size $\eta$ in the bulk so that the typical number of eigenvalues is of order $N \eta$. In the usual statement of the semicircle law, $\eta$ is a fixed number independent of $N$ and it is taken to zero only after the limit $N \rightarrow \infty$. This can be viewed as the largest scale on which the semicircle law is valid. On the other extreme, for the smallest scale, one may take $\eta=k / N$ and take the limit $N \rightarrow \infty$ followed by $k \rightarrow \infty$. If the semicircle law is valid in this sense, we shall say that the local semicircle law holds. Below this smallest scale, the eigenvalue distribution is expected to be governed by the Dyson statistics related to sine kernels. The Dyson statistics was proved for many ensembles (see [1,4] for a review), including Wigner matrices with Gaussian convoluted distributions [7].

In this paper, we establish the local semicircle law up to logarithmic factors in the energy scale, i.e., for $\eta \sim N^{-1}(\log N)^{8}$. The result holds for any energy window in the bulk spectrum away from the spectral edges. In [5] we have proved the same statement for $\eta \gg N^{-2 / 3}$ (modulo logarithmic corrections). Prior to our work the best result was obtained in [2] for $\eta \gg N^{-1 / 2}$. See also [6] and [8] for related and earlier results. As a corollary, our result also proves that no gap between consecutive bulk eigenvalues can be bigger than $C(\log N)^{8} / N$, to be compared with the expected average $1 / N$ behavior given by Dyson's law.

It is widely believed that the eigenvalue distribution of the Wigner random matrix and the random Schrödinger operator in the extended (or delocalized) state regime are the same up to normalizations. Although this conjecture is far from the reach of the current method, a natural question arises as to whether the eigenvectors of random matrices are extended. More precisely, if $\mathbf{v}=\left(v_{1}, \ldots, v_{N}\right)$ is an $\ell^{2}$-normalized eigenvector, $\|\mathbf{v}\|=1$, we say that $\mathbf{v}$ is completely delocalized if $\|\mathbf{v}\|_{\infty}=\max _{j}\left|v_{j}\right|$ is bounded from above by $C N^{-1 / 2}$, the average size of $\left|v_{j}\right|$. In this paper, we shall prove that all eigenvectors with eigenvalues away from the spectral edges are completely delocalized (modulo logarithmic corrections) in probability. Similar results, but with $C N^{-1 / 2}$ replaced by $C N^{-1 / 3}$ were proved in [5]. Notice that our new result, in particular, answers (up to logarithmic factors) the question posed by T. Spencer whether $\|\mathbf{v}\|_{4}$ is of order $N^{-1 / 4}$.

Denote the $(i, j)$-th entry of an $N \times N$ matrix $H$ by $h_{i, j}=h_{i j}$. When there is no confusion, we omit the comma between the two subscripts. We shall assume that the matrix is Hermitian, i.e., $h_{i j}=\overline{h_{j i}}$. These matrices form a Hermitian Wigner ensemble if

$$
h_{i j}=N^{-1 / 2}\left[x_{i j}+\sqrt{-1} y_{i j}\right], \quad(i<j), \quad \text { and } \quad h_{i i}=N^{-1 / 2} x_{i i},
$$

where $x_{i j}, y_{i j}(i<j)$ and $x_{i i}$ are independent real random variables with mean zero. We assume that $x_{i j}, y_{i j}(i<j)$ all have a common distribution $\nu$ with variance $1 / 2$ and with a strictly positive density function: $\mathrm{d} \nu(x)=$ (const.) $e^{-g(x)} \mathrm{d} x$. The diagonal elements, $x_{i i}$, also have a common distribution, $\mathrm{d} \widetilde{\nu}(x)=$ (const.) $e^{-\widetilde{g}(x)} \mathrm{d} x$, that may be different from $\mathrm{d} \nu$. Let $\mathbb{P}$ and $\mathbb{E}$ denote the probability and the expectation value, respectively, w.r.t the joint distribution of all matrix elements.

We need to assume further conditions on the distributions of the matrix elements in addition to (1.1).

C1) The function $g$ is twice differentiable and it satisfies

$$
\sup _{x \in \mathbb{R}} g^{\prime \prime}(x)<\infty .
$$


C2) There exists a $\delta>0$ such that

$$
\int e^{\delta x^{2}} \mathrm{~d} \widetilde{\nu}(x)<\infty .
$$

C4) The measure $\nu$ satisfies the logarithmic Sobolev inequality, i.e., there exists a constant $C_{\text {sob }}$ such that for any density function $u>0$ with $\int u \mathrm{~d} \nu=1$,

$$
\int u \log u \mathrm{~d} \nu \leq C_{\text {sob }} \int|\nabla \sqrt{u}|^{2} \mathrm{~d} \nu .
$$

Here we have followed the convention in [5] to use the label C4) for the logarithmic Sobolev bound and reserved C3) for a spectral gap condition in [5]. We will also need the decay condition (1.3) for the measure d $\nu$, i.e., for some small $\delta>0$,

$$
\int e^{\delta x^{2}} \mathrm{~d} \nu(x)<\infty .
$$

This condition was assumed in the earlier version of the manuscript, but J.-D. Deuschel and M. Ledoux kindly pointed out to us that (1.5) follows from C4), see [9].

Condition C1) is needed only because we will use Lemma 2.3 of [5] in the proof of the following Theorem 1.1. J. Bourgain has informed us that this lemma can also be proved without this condition. We include the precise statement and his proof in the Appendix.

Notation. We will use the notation $|A|$ both for the Lebesgue measure of a set $A \subset \mathbb{R}$ and for the cardinality of a discrete set $A \subset \mathbb{Z}$. The usual Hermitian scalar product for vectors $\mathbf{x}, \mathbf{y} \in \mathbb{C}^{N}$ will be denoted by $\mathbf{x} \cdot \mathbf{y}$ or by $(\mathbf{x}, \mathbf{y})$. We will use the convention that $C$ denotes generic large constants and $c$ denotes generic small positive constants whose values may change from line to line. Since we are interested in large matrices, we always assume that $N$ is sufficiently large.

Let $H$ be the $N \times N$ Wigner matrix with eigenvalues $\mu_{1} \leq \mu_{2} \leq \ldots \leq \mu_{N}$. For any spectral parameter $z=E+i \eta \in \mathbb{C}, \eta>0$, we denote the Green function by $G_{z}=(H-z)^{-1}$. Let $F(x)=F_{N}(x)$ be the empirical distribution function of the eigenvalues

$$
F(x)=\frac{1}{N}\left|\left\{\alpha: \mu_{\alpha} \leq x\right\}\right| .
$$

We define the Stieltjes transform of $F$ as

$$
m=m(z)=\frac{1}{N} \operatorname{Tr} G_{z}=\int_{\mathbb{R}} \frac{\mathrm{d} F(x)}{x-z},
$$

and we let

$$
\rho=\rho_{\eta}(E)=\frac{\operatorname{Im} m(z)}{\pi}=\frac{1}{N \pi} \operatorname{Im} \operatorname{Tr} G_{z}=\frac{1}{N \pi} \sum_{\alpha=1}^{N} \frac{\eta}{\left(\mu_{\alpha}-E\right)^{2}+\eta^{2}}
$$

be the normalized density of states of $H$ around energy $E$ and regularized on scale $\eta$. The random variables $m$ and $\varrho$ also depend on $N$, when necessary, we will indicate this fact by writing $m_{N}$ and $\varrho_{N}$.

For any $z=E+i \eta$ we let

$$
m_{s c}=m_{s c}(z)=\int_{\mathbb{R}} \frac{\varrho_{s c}(x) \mathrm{d} x}{x-z}
$$


be the Stieltjes transform of the Wigner semicircle distribution function whose density is given by

$$
\varrho_{s c}(x)=\frac{1}{2 \pi} \sqrt{4-x^{2}} \mathbf{1}(|x| \leq 2) .
$$

For $\kappa, \widetilde{\eta}>0$ we define the set

$$
S_{N, \kappa, \widetilde{\eta}}:=\{z=E+i \eta \in \mathbb{C}:|E| \leq 2-\kappa, \widetilde{\eta} \leq \eta \leq 1\}
$$

and for $\widetilde{\eta}=N^{-1}(\log N)^{8}$ we write

$$
S_{N, \kappa}:=\left\{z=E+i \eta \in \mathbb{C}:|E| \leq 2-\kappa, \frac{(\log N)^{8}}{N} \leq \eta \leq 1\right\} .
$$

The following two theorems are the main results of this paper.

Theorem 1.1 Let $H$ be an $N \times N$ Wigner matrix as described in (1.1) and assume the conditions (1.2), (1.3) and (1.4). Then for any $\kappa>0$ and $\varepsilon>0$, the Stieltjes transform $m_{N}(z)$ (see (1.7)) of the empirical eigenvalue distribution of the $N \times N$ Wigner matrix satisfies

$$
\mathbb{P}\left\{\sup _{z \in S_{N, \kappa}}\left|m_{N}(z)-m_{s c}(z)\right| \geq \varepsilon\right\} \leq e^{-c(\log N)^{2}}
$$

where $c>0$ depends on $\kappa, \varepsilon$. In particular, the density of states $\varrho_{\eta}(E)$ converges to the Wigner semicircle law in probability uniformly for all energies away from the spectral edges and for all energy windows at least $N^{-1}(\log N)^{8}$.

Furthermore, let $\eta^{*}=\eta^{*}(N)$ such that $(\log N)^{8} / N \ll \eta^{*} \ll 1$ as $N \rightarrow \infty$, then we have the convergence of the counting function as well:

$$
\mathbb{P}\left\{\sup _{|E| \leq 2-\kappa}\left|\frac{\mathcal{N}_{\eta^{*}}(E)}{2 N \eta^{*}}-\varrho_{s c}(E)\right| \geq \varepsilon\right\} \leq e^{-c(\log N)^{2}}
$$

for any $\varepsilon>0$, where $\mathcal{N}_{\eta^{*}}(E)=\left|\left\{\alpha:\left|\mu_{\alpha}-E\right| \leq \eta^{*}\right\}\right|$ denotes the number of eigenvalues in the interval $\left[E-\eta^{*}, E+\eta^{*}\right]$.

This result identifies the density of states away from the spectral edges in a window where the typical number of eigenvalues is of order bigger than $(\log N)^{8}$. Our scale is not sufficiently small to identify individual eigenvalues, in particular we do not know whether the local eigenvalue spacing follows the expected Dyson statistics characterized by the sine-kernel or some other local statistics, e.g., that of a Poisson point process.

Theorem 1.2 Let $H$ be an $N \times N$ Wigner matrix as described in (1.1) and satisfying the conditions (1.2), (1.3) and (1.4). Fix $\kappa>0$, and assume that $C$ is large enough. Then there exists $c>0$ such that

$$
\mathbb{P}\left\{\exists \mathbf{v} \text { with } H \mathbf{v}=\mu \mathbf{v},\|\mathbf{v}\|=1, \mu \in[-2+\kappa, 2-\kappa] \text { and }\|\mathbf{v}\|_{\infty} \geq \frac{C(\log N)^{9 / 2}}{N^{1 / 2}}\right\} \leq e^{-c(\log N)^{2}} .
$$

We now sketch the key idea to prove Theorem 1.1; Theorem 1.2 can be proved following similar ideas used in [5]. 
Let $B^{(k)}$ denote the $(N-1) \times(N-1)$ minor of $H$ after removing the $k$-th row and $k$-th column and let $m_{k}(z)$ denote the Stieltjes transform of the eigenvalue distribution function associated with $B^{(k)}$. It is known that $m(z)$, defined in (1.7), satisfies a recurrence relation

$$
m(z)=\frac{1}{N} \sum_{k=1}^{N} \frac{1}{h_{k k}-z-\left(1-\frac{1}{N}\right) m^{(k)}(z)-X_{k}},
$$

where $X_{k}$ (defined precisely in (2.4)) is an "error" term depending on $B^{(k)}$ and the $k$-th column and row elements of the random matrix $H$. If we neglect $X_{k}$ (and $h_{k k}$ which is of order $N^{-1 / 2}$ by definition) and replace $m^{(k)}$ by $m$, we obtain an equation for $m$ and this leads to the Stieltjes transform of the semi-circle law. So our main task is to prove that $X_{k}$ is negligible. Unfortunately, $X_{k}$ depends crucially on the eigenvalues and eigenfunctions of $B^{(k)}$. In an earlier work [2], the estimate on $X_{k}$ was done via an involved bootstrap argument (and valid up to order $N^{-1 / 2}$ ). The bootstrapping is needed in [2] since $X_{k}$ depends critically on properties of $B^{(k)}$ for which there was only limited a priori information. In our preceding paper [5], we split $m$ and $m^{(k)}$ into their means and variances; the variances were then shown to be negligible up to the scale $N^{-2 / 3}$ (The variance control of $m$ up to the scale $N^{-1 / 2}$ was already in [6]). On the other hand, the means of $m$ and $m^{(k)}$ are very close due to the fact that the eigenvalues of $H$ and $B^{(k)}$ are interlaced. Finally, $X_{k}$ was controlled via an estimate on its fourth moment. We have thus arrived at a fixed point equation for the mean of $m$ whose unique solution is the Stieltjes transform of the semi-circle law.

In the current paper, we avoid the variance control by viewing $m$ and $m^{(k)}$ directly as random variables in the recurrence relation (1.11). Furthermore, the moment control on $X_{k}$ is now improved to an exponential moment estimate. Since our estimate on the fourth moment of $X_{k}$ was done via a spectral gap argument, it is a folklore that moment estimates usually can be lifted to an exponential moment estimate provided the spectral gap estimate is replaced by a logarithmic Sobolev inequality. In this paper, we use a concentration of measure inequality to avoid all bootstrap arguments appearing both in [2] and [8]. In the previous version of this paper we obtained the concentration inequality by using the logarithmic Sobolev inequality together with the Gibbs entropy inequality. We would like to thank the referee who pointed out to us that the concentration inequality of Bobkov and Götze [3] can be used directly to shortcut our original proof. The applicability of the Bobkov-Götze inequality as well as some of the heuristic arguments presented here, however, depend crucially on an a priori upper bound on $|m(z)|$; this was obtained via a large deviation estimate on the eigenvalue concentration [5].

\section{Proof of Theorem 1.1}

The proof of (1.10) follows from (1.9) exactly as in Corollary 4.2 of [5], so we focus on proving (1.9). We first remove the supremum in (1.9).

For any two points $z, z^{\prime} \in S_{N, \kappa, \eta}$ we have

$$
\left|m_{N}(z)-m_{N}\left(z^{\prime}\right)\right| \leq N^{2}\left|z-z^{\prime}\right|
$$

since the gradient of $m_{N}(z)$ is bounded by $|\operatorname{Im} z|^{-2} \leq N^{2}$ on $S_{N, \kappa}$. We can choose a set of at most $Q=C \varepsilon^{-2} N^{4}$ points, $z_{1}, z_{2}, \ldots, z_{Q}$, in $S_{N, \kappa, \eta}$ such that for any $z \in S_{N, \kappa, \eta}$ there exists a point $z_{j}$ with $\left|z-z_{j}\right| \leq \frac{1}{4} \varepsilon N^{-2}$. In particular, $\left|m_{N}(z)-m_{N}\left(z_{j}\right)\right| \leq \varepsilon / 4$ if $N$ is large enough and $\left|m_{s c}(z)-m_{s c}\left(z_{j}\right)\right| \leq \varepsilon / 4$. Since $\operatorname{Im} z_{j} \geq \eta$, under the condition that $\eta \geq N^{-1}(\log N)^{8}$ we have

$$
\mathbb{P}\left\{\sup _{z \in S_{N, \kappa}}\left|m_{N}(z)-m_{s c}(z)\right| \geq \varepsilon\right\} \leq \sum_{j=1}^{Q} \mathbb{P}\left\{\left|m_{N}\left(z_{j}\right)-m_{s c}\left(z_{j}\right)\right| \geq \frac{\varepsilon}{2}\right\}
$$


Therefore, in order to conclude (1.9), it suffices to prove that

$$
\mathbb{P}\left\{\left|m_{N}(z)-m_{s c}(z)\right| \geq \varepsilon\right\} \leq e^{-c(\log N)^{2}}
$$

for each fixed $z \in S_{N, \kappa}$.

Let $B^{(k)}$ denote the $(N-1) \times(N-1)$ minor of $H$ after removing the $k$-th row and $k$-th column. Note that $B^{(k)}$ is an $(N-1) \times(N-1)$ Hermitian Wigner matrix with a normalization factor off by $\left(1-\frac{1}{N}\right)^{1 / 2}$. Let $\lambda_{1}^{(k)} \leq \lambda_{2}^{(k)} \leq \ldots \leq \lambda_{N-1}^{(k)}$ denote its eigenvalues and $\mathbf{u}_{1}^{(k)}, \ldots, \mathbf{u}_{N-1}^{(k)}$ the corresponding normalized eigenvectors.

Let $\mathbf{a}^{(k)}=\left(h_{k, 1}, h_{k, 2}, \ldots h_{k, k-1}, h_{k, k+1}, \ldots h_{k, N}\right)^{*} \in \mathbb{C}^{N-1}$, i.e. the $k$-th column after removing the diagonal element $h_{k, k}=h_{k k}$. Computing the $(k, k)$ diagonal element of the resolvent $G_{z}$, we have

$$
G_{z}(k, k)=\frac{1}{h_{k k}-z-\mathbf{a}^{(k)} \cdot\left(B^{(k)}-z\right)^{-1} \mathbf{a}^{(k)}}=\left[h_{k k}-z-\frac{1}{N} \sum_{\alpha=1}^{N-1} \frac{\xi_{\alpha}^{(k)}}{\lambda_{\alpha}^{(k)}-z}\right]^{-1}
$$

where we defined

$$
\xi_{\alpha}^{(k)}:=\left|\sqrt{N} \mathbf{a}^{(k)} \cdot \mathbf{u}_{\alpha}^{(k)}\right|^{2} .
$$

Similarly to the definition of $m(z)$ in (1.7), we also define the Stieltjes transform of the density of states of $B^{(k)}$

$$
m^{(k)}=m^{(k)}(z)=\frac{1}{N-1} \operatorname{Tr} \frac{1}{B^{(k)}-z}=\int_{\mathbb{R}} \frac{\mathrm{d} F^{(k)}(x)}{x-z}
$$

with the empirical counting function

$$
F^{(k)}(x)=\frac{1}{N-1}\left|\left\{\alpha: \lambda_{\alpha}^{(k)} \leq x\right\}\right| .
$$

The spectral parameter $z$ is fixed throughout the proof and we will often omit it from the argument of the Stieltjes transforms.

It follows from (2.2) that

$$
m=m(z)=\frac{1}{N} \sum_{k=1}^{N} G_{z}(k, k)=\frac{1}{N} \sum_{k=1}^{N} \frac{1}{h_{k k}-z-\mathbf{a}^{(k)} \cdot\left(B^{(k)}-z\right)^{-1} \mathbf{a}^{(k)}} .
$$

Let $\mathbb{E}_{k}$ denote the expectation value w.r.t the random vector $\mathbf{a}^{(k)}$. Define the random variable

$$
X_{k}(z)=X_{k}:=\mathbf{a}^{(k)} \cdot \frac{1}{B^{(k)}-z} \mathbf{a}^{(k)}-\mathbb{E}_{k} \mathbf{a}^{(k)} \cdot \frac{1}{B^{(k)}-z} \mathbf{a}^{(k)}=\frac{1}{N} \sum_{\alpha=1}^{N-1} \frac{\xi_{\alpha}^{(k)}-1}{\lambda_{\alpha}^{(k)}-z}
$$

where we used that $\mathbb{E}_{k} \xi_{\alpha}^{(k)}=\left\|\mathbf{u}_{\alpha}^{(k)}\right\|^{2}=1$.

We note that

$$
\mathbb{E}_{k} \mathbf{a}^{(k)} \cdot \frac{1}{B^{(k)}-z} \mathbf{a}^{(k)}=\frac{1}{N} \sum_{\alpha} \frac{1}{\lambda_{\alpha}^{(k)}-z}=\left(1-\frac{1}{N}\right) m^{(k)}
$$

With this notation it follows from (2.2) that

$$
m=\frac{1}{N} \sum_{k=1}^{N} \frac{1}{h_{k k}-z-\left(1-\frac{1}{N}\right) m^{(k)}-X_{k}} .
$$


We use that

$$
\left|m-\left(1-\frac{1}{N}\right) m^{(k)}\right|=\left|\int \frac{\mathrm{d} F(x)}{x-z}-\left(1-\frac{1}{N}\right) \int \frac{\mathrm{d} F^{(k)}(x)}{x-z}\right|=\frac{1}{N}\left|\int \frac{N F(x)-(N-1) F^{(k)}(x)}{(x-z)^{2}} \mathrm{~d} x\right| .
$$

We recall that the eigenvalues of $H$ and $B^{(k)}$ are interlaced,

$$
\mu_{1} \leq \lambda_{1}^{(k)} \leq \mu_{2} \leq \lambda_{2}^{(k)} \leq \ldots \leq \lambda_{N-1}^{(k)} \leq \mu_{N},
$$

(see e.g. Lemma 2.5 of [5]), therefore we have $\max _{x}\left|N F(x)-(N-1) F^{(k)}(x)\right| \leq 1$. Thus

$$
\left|m-\left(1-\frac{1}{N}\right) m^{(k)}\right| \leq \frac{1}{N} \int \frac{\mathrm{d} x}{|x-z|^{2}} \leq \frac{C}{N \eta} .
$$

We postpone the proof of the following lemma:

Lemma 2.1 Suppose that $\mathbf{v}_{\alpha}$ and $\lambda_{\alpha}$ are eigenvectors and eigenvalues of an $N \times N$ random matrix with a law satisfying the assumption of Theorem 1.1. Let

$$
X=\frac{1}{N} \sum_{\alpha} \frac{\xi_{\alpha}-1}{\lambda_{\alpha}-z}
$$

with $z=E+i \eta, \xi_{\alpha}=\left|\mathbf{b} \cdot \mathbf{v}_{\alpha}\right|^{2}$, where the components of $\mathbf{b}$ are i.i.d. random variables satisfying (1.4). Then there exist sufficiently small positive constants $\varepsilon_{0}$ and $c$ such that in the joint product probability space of $\mathbf{b}$ and the law of the random matrices we have

$$
\mathbb{P}[|X| \geq \varepsilon] \leq e^{-c \varepsilon(\log N)^{2}}
$$

for any $\varepsilon \leq \varepsilon_{0}$ and $\eta \geq(\log N)^{8} / N$.

For a given $\varepsilon>0$ and $z=E+i \eta \in S_{N, \kappa}$, we set $z_{n}=E+i 2^{n} \eta$ and we define the event

$$
\Omega=\bigcup_{k=1}^{N} \bigcup_{n=0}^{\left[\log _{2}(1 / \eta)\right]}\left\{\left|X_{k}\left(z_{n}\right)\right| \geq \varepsilon / 3\right\} \cup \bigcup_{k=1}^{N}\left\{\left|h_{k k}\right| \geq \varepsilon / 3\right\},
$$

where $[\cdot]$ denotes the integer part. Since $h_{k k}=N^{-1 / 2} b_{k k}$ with $b_{k k}$ satisfying (1.3), we have

$$
\mathbb{P}\left\{\left|h_{k k}\right| \geq \varepsilon / 3\right\} \leq C e^{-\delta \varepsilon^{2} N / 9} .
$$

We now apply Lemma 2.1 for each $X_{k}\left(z_{n}\right)$ and conclude that

$$
\mathbb{P}(\Omega) \leq e^{-c \varepsilon(\log N)^{2}}
$$

with a sufficiently small $c>0$.

On the complement $\Omega^{c}$ we have from (2.5)

$$
m\left(z_{n}\right)=\frac{1}{N} \sum_{k=1}^{N} \frac{1}{-m\left(z_{n}\right)-z_{n}+\delta_{k}}
$$


where

$$
\delta_{k}=\delta_{k}\left(z_{n}\right)=h_{k k}+m\left(z_{n}\right)-\left(1-\frac{1}{N}\right) m_{k}\left(z_{n}\right)-X_{k}\left(z_{n}\right)
$$

are random variables satisfying $\left|\delta_{k}\right| \leq \varepsilon$ by (2.7). After expansion, the last equation implies that

$$
\left|m\left(z_{n}\right)+\frac{1}{m\left(z_{n}\right)+z_{n}}\right| \leq \frac{\varepsilon}{\left(\operatorname{Im}\left(m\left(z_{n}\right)+z_{n}\right)\right)\left(\operatorname{Im}\left(m\left(z_{n}\right)+z_{n}\right)-\varepsilon\right)},
$$

if $\operatorname{Im}\left(m\left(z_{n}\right)+z_{n}\right)>\varepsilon$, using that $\left|-m\left(z_{n}\right)-z_{n}+\delta_{k}\right| \geq \operatorname{Im}\left(m\left(z_{n}\right)+z_{n}\right)-\varepsilon$.

We note that for any $z \in S_{N, \kappa}$ the equation

$$
M+\frac{1}{M+z}=0
$$

has a unique solution with $\operatorname{Im} M>0$, namely $M=m_{s c}(z)$, the Stieltjes transform of the semicircle law. Note that there exists $c(\kappa)>0$ such that $\operatorname{Im} m_{s c}(E+i \eta) \geq c(\kappa)$ for any $|E| \leq 2-\kappa$, uniformly in $\eta$.

The equation (2.9) is stable in the following sense. For any small $\delta$, let $M=M(z, \delta)$ be a solution to

$$
M+\frac{1}{M+z}=\delta
$$

with $\operatorname{Im} M>0$. Explicitly, we have

$$
M=\frac{-z+\sqrt{z^{2}-4+2 z \delta+\delta^{2}}}{2}+\frac{\delta}{2}
$$

where we have chosen the square root so that $\operatorname{Im} M>0$ when $\delta=0$ and $\operatorname{Im} z>0$. On the compact set $z \in S_{N, \kappa},\left|z^{2}-4\right|$ is bounded away from zero and thus

$$
\left|M-m_{s c}\right| \leq C_{\kappa} \delta
$$

for some constant $C_{\kappa}$ depending only on $\kappa$.

Now we perform a bootstrap argument in the imaginary part of $z$ to prove that

$$
\left|m(z)-m_{s c}(z)\right| \leq C^{*} \varepsilon
$$

uniformly in $z \in S_{N, \kappa}$ with a sufficiently large constant $C^{*}$. Fix $z=E+i \eta$ with $|E| \leq 2-\kappa$ and let $z_{n}=E+i 2^{n} \eta$. For $n=\left[\log _{2}(1 / \eta)\right]$, we have $\operatorname{Im} z_{n} \in\left[\frac{1}{2}, 1\right],(2.12)$ follows from (2.8) with some small $\varepsilon$, since the right hand side of (2.8) is bounded by $C \varepsilon$. Suppose now that (2.12) has been proven for $z=z_{n}$, for some $n \geq 1$ with $\eta_{n}=\operatorname{Im} z_{n} \in\left[2 N^{-1}(\log N)^{8}, 1\right]$. We want to prove it for $z=z_{n-1}$, with $\operatorname{Im} z_{n-1}=\eta_{n} / 2$. By integrating the inequality

$$
\frac{\eta_{n} / 2}{(x-E)^{2}+\left(\eta_{n} / 2\right)^{2}} \geq \frac{1}{2} \frac{\eta_{n}}{(x-E)^{2}+\eta_{n}^{2}}
$$

with respect to $\mathrm{d} F(x)$ we obtain that

$$
\operatorname{Im} m\left(z_{n-1}\right) \geq \frac{1}{2} \operatorname{Im} m\left(z_{n}\right) \geq \frac{1}{2} c(\kappa)-C^{*} \varepsilon>\frac{c(\kappa)}{4}
$$

for sufficiently small $\varepsilon$, where (2.12) and $\operatorname{Im} m_{s c}\left(z_{n}\right) \geq c(\kappa)$ were used. Thus the right hand side of (2.8) with $z_{n}$ replaced with $z_{n-1}$ is bounded by $C \varepsilon$, the constant depending only on $\kappa$. Applying the stability bound (2.11), we get (2.12) for $z=z_{n-1}$. Continuing the induction argument, finally we obtain (2.12) for $z=z_{0}=E+i \eta$. 


\section{$3 \quad$ Proof of Lemma 2.1}

Let $I_{n}=[n \eta,(n+1) \eta]$ and $K_{0}$ be a sufficiently large number. We have $\left[-K_{0}, K_{0}\right] \subset \cup_{n=-m}^{m} I_{n}$ with $m \leq C K_{0} / \eta$. Denote by $\Omega$ the event

$$
\Omega:=\left\{\max _{n} \mathcal{N}_{I_{n}} \geq N \eta(\log N)^{2}\right\} \cup\left\{\max _{\alpha}\left|\lambda_{\alpha}\right| \geq K_{0}\right\}
$$

where $\mathcal{N}_{I_{n}}=\left|\left\{\alpha: \lambda_{\alpha} \in I_{n}\right\}\right|$ is the number of eigenvalues in the interval $I_{n}$. From Theorem 2.1 and Lemma 7.4 of [5], the probability of $\Omega$ is bounded by

$$
\mathbb{P}(\Omega) \leq e^{-c(\log N)^{2}}
$$

for some sufficiently small $c>0$. Therefore, if $\mathbb{P}_{\mathbf{b}}$ denotes the probability w.r.t. the variable $\mathbf{b}$, we find

$$
\begin{aligned}
\mathbb{P}[|X| \geq \varepsilon] \leq & e^{-c(\log N)^{2}}+\mathbb{E}\left[\mathbf{1}_{\Omega^{c}} \mathbb{P}_{\mathbf{b}}[|X| \geq \varepsilon]\right] \\
\leq & e^{-c(\log N)^{2}}+\mathbb{E}\left[\mathbf{1}_{\Omega^{c}} \cdot \mathbb{P}_{\mathbf{b}}[\operatorname{Re} X \leq-\varepsilon / 2]\right]+\mathbb{E}\left[\mathbf{1}_{\Omega^{c}} \cdot \mathbb{P}_{\mathbf{b}}[\operatorname{Re} X \geq \varepsilon / 2]\right] \\
& \quad+\mathbb{E}\left[\mathbf{1}_{\Omega^{c}} \cdot \mathbb{P}_{\mathbf{b}}[\operatorname{Im} X \leq-\varepsilon / 2]\right]+\mathbb{E}\left[\mathbf{1}_{\Omega^{c}} \cdot \mathbb{P}_{\mathbf{b}}[\operatorname{Im} X \geq \varepsilon / 2]\right]
\end{aligned}
$$

The last four terms on the r.h.s. of the last equation can all be handled with similar arguments; we show, for example, how to bound the last term. For any $T>0$, we have

$$
\mathbb{E}\left[\mathbf{1}_{\Omega^{c}} \cdot \mathbb{P}_{\mathbf{b}}[\operatorname{Im} X \geq \varepsilon / 2]\right] \leq e^{-T \varepsilon / 2} \mathbb{E}\left[\mathbf{1}_{\Omega^{c}} \mathbb{E}_{\mathbf{b}} e^{T \operatorname{Im} X}\right]
$$

where $\mathbb{E}_{\mathbf{b}}$ denotes the expectation w.r.t. the variable $\mathbf{b}$. Using the fact that the distribution of the components of $\mathbf{b}$ satisfies the log-Sobolev inequality (1.4), it follows from the concentration inequality (Theorem 2.1 from [3]) that

$$
\mathbb{E}_{\mathbf{b}} e^{T \operatorname{Im} X} \leq \mathbb{E}_{\mathbf{b}} \exp \left(\frac{C_{\mathrm{sob}} T^{2}}{2}|\nabla(\operatorname{Im} X)|^{2}\right)
$$

where

$$
\begin{aligned}
|\nabla(\operatorname{Im} X)|^{2} & =\sum_{k}\left(\left|\frac{\partial(\operatorname{Im} X)}{\partial\left(\operatorname{Re} b_{k}\right)}\right|^{2}+\left|\frac{\partial(\operatorname{Im} X)}{\partial\left(\operatorname{Im} b_{k}\right)}\right|^{2}\right) \\
& =\sum_{k}\left(\left|\frac{\eta}{N} \sum_{\alpha} \frac{1}{\left|\lambda_{\alpha}-z\right|^{2}}\left(\left(\mathbf{b} \cdot \mathbf{v}_{\alpha}\right) \overline{\mathbf{v}}_{\alpha}(k)+\left(\overline{\mathbf{b} \cdot \mathbf{v}_{\alpha}}\right) \mathbf{v}_{\alpha}(k)\right)\right|^{2}\right. \\
& \left.\quad+\left|\frac{\eta}{N} \sum_{\alpha} \frac{1}{\left|\lambda_{\alpha}-z\right|^{2}}\left(\left(\mathbf{b} \cdot \mathbf{v}_{\alpha}\right) \overline{\mathbf{v}}_{\alpha}(k)-\left(\overline{\mathbf{b} \cdot \mathbf{v}_{\alpha}}\right) \mathbf{v}_{\alpha}(k)\right)\right|^{2}\right) \\
& =4 \frac{\eta^{2}}{N^{2}} \sum_{\alpha} \frac{\xi_{\alpha}}{\left|\lambda_{\alpha}-z\right|^{4}} \\
& \leq \frac{4}{N \eta} Y .
\end{aligned}
$$

Here we defined the random variable

$$
Y=\frac{1}{N} \sum_{\alpha} \frac{\xi_{\alpha}}{\left|\lambda_{\alpha}-z\right|}
$$


From $(3.2)$, choosing $T=2(\log N)^{2}$ and using that $N \eta \geq(\log N)^{8}$, we obtain

$$
\mathbb{E}\left[\mathbf{1}_{\Omega^{c}} \cdot \mathbb{P}_{\mathbf{b}}[\operatorname{Im} X \geq \varepsilon / 2]\right] \leq e^{-\varepsilon(\log N)^{2}} \mathbb{E}\left[\mathbf{1}_{\Omega^{c}} \mathbb{E}_{\mathbf{b}} \exp \left(\frac{8 C_{\text {sob }}}{(\log N)^{4}} Y\right)\right] .
$$

Let $\nu=8 C_{\text {sob }} /(\log N)^{4}$. By Hölder inequality, we can estimate

$$
\mathbb{E}_{\mathbf{b}} e^{\nu Y}=\mathbb{E}_{\mathbf{b}} \prod_{\alpha} \exp \left[\frac{\nu}{N\left|\lambda_{\alpha}-z\right|} \xi_{\alpha}\right] \leq \prod_{\alpha}\left(\mathbb{E}_{\mathbf{b}} \exp \left[\frac{\nu c_{\alpha}}{N\left|\lambda_{\alpha}-z\right|} \xi_{\alpha}\right]\right)^{1 / c_{\alpha}}
$$

where $\sum_{\alpha} \frac{1}{c_{\alpha}}=1$. We shall choose

$$
c_{\alpha}=\varrho \frac{N\left|\lambda_{\alpha}-z\right|}{\nu}
$$

where $\varrho$ is given by

$$
\varrho=\frac{\nu}{N} \sum_{\alpha} \frac{1}{\left|\lambda_{\alpha}-z\right|} \leq \frac{\nu \log N}{N \eta} \max _{n} \mathcal{N}_{I_{n}} \leq \nu(\log N)^{3}=\frac{8 C_{\text {sob }}}{\log N} .
$$

Here we have used $\max _{n} \mathcal{N}_{I_{n}} \leq N \eta(\log N)^{2}$ due to that we are in the set $\Omega^{c}$. Notice that with this choice,

$$
\frac{\nu c_{\alpha}}{N\left|\lambda_{\alpha}-z\right|} \leq \frac{8 C_{\mathrm{sob}}}{\log N}
$$

is a small number. In the proof of Lemma 7.4 of [5] (see equation (7.13) of [5]) we showed that

$$
\mathbb{E}_{\mathbf{b}} e^{\tau \xi_{\alpha}}<K
$$

with a universal constant $K$ if $\tau$ is sufficiently small depending on $\delta$ in (1.3). From (3.5), it follows that

$$
\mathbb{E}\left[\mathbf{1}_{\Omega^{c}} \cdot \mathbb{P}_{\mathbf{b}}[\operatorname{Im} X \geq \varepsilon / 2]\right] \leq K e^{-\varepsilon(\log N)^{2}}
$$

Since similar bounds hold for the other terms on the r.h.s. of (3.1) as well, this concludes the proof of the lemma.

\section{Delocalization of eigenvectors}

Here we prove Theorem 1.2, the argument follows the same line as in [5] (Proposition 5.3). Let $\eta^{*}=$ $N^{-1}(\log N)^{9}$ and partition the interval $[-2+\kappa, 2-\kappa]$ into $n_{0}=O\left(1 / \eta^{*}\right) \leq O(N)$ intervals $I_{1}, I_{2}, \ldots I_{n_{0}}$ of length $\eta^{*}$. As before, let $\mathcal{N}_{I}=\left|\left\{\beta: \mu_{\beta} \in I\right\}\right|$ denote the eigenvalues in $I$. By using (1.10) in Theorem 1.1, we have

$$
\mathbb{P}\left\{\max _{n} \mathcal{N}_{I_{n}} \leq \varepsilon N \eta^{*}\right\} \leq e^{-c(\log N)^{2}}
$$

if $\varepsilon$ is sufficiently small (depending on $\kappa$ ). Suppose that $\mu \in I_{n}$, and that $H \mathbf{v}=\mu \mathbf{v}$. Consider the decomposition

$$
H=\left(\begin{array}{cc}
h & \mathbf{a}^{*} \\
\mathbf{a} & B
\end{array}\right)
$$


where $\mathbf{a}=\left(h_{1,2}, \ldots h_{1, N}\right)^{*}$ and $B$ is the $(N-1) \times(N-1)$ matrix obtained by removing the first row and first column from $H$. Let $\lambda_{\alpha}$ and $\mathbf{u}_{\alpha}$ (for $\alpha=1,2, \ldots, N-1$ ) denote the eigenvalues and the normalized eigenvectors of $B$. From the eigenvalue equation $H \mathbf{v}=\mu \mathbf{v}$ and from (4.1) we find that

$$
h v_{1}+\mathbf{a} \cdot \mathbf{w}=\mu v_{1}, \quad \text { and } \quad \mathbf{a} v_{1}+B \mathbf{w}=\mu \mathbf{w}
$$

with $\mathbf{w}=\left(v_{2}, \ldots, v_{N}\right)^{t}$. From these equations we obtain $\mathbf{w}=(\mu-B)^{-1} \mathbf{a} v_{1}$ and thus

$$
\|\mathbf{w}\|^{2}=\mathbf{w} \cdot \mathbf{w}=\left|v_{1}\right|^{2} \mathbf{a} \cdot(\mu-B)^{-2} \mathbf{a}
$$

Since $\|\mathbf{w}\|^{2}=1-\left|v_{1}\right|^{2}$, we obtain

$$
\left|v_{1}\right|^{2}=\frac{1}{1+\mathbf{a} \cdot(\mu-B)^{-2} \mathbf{a}}=\frac{1}{1+\frac{1}{N} \sum_{\alpha} \frac{\xi_{\alpha}}{\left(\mu-\lambda_{\alpha}\right)^{2}}} \leq \frac{4 N\left[\eta^{*}\right]^{2}}{\sum_{\lambda_{\alpha} \in I_{n}} \xi_{\alpha}},
$$

where in the second equality we set $\xi_{\alpha}=\left|\sqrt{N} \mathbf{a} \cdot \mathbf{u}_{\alpha}\right|^{2}$ and used the spectral representation of $B$. By the interlacing property of the eigenvalues of $H$ and $B$, there exist at least $\mathcal{N}_{I_{n}}-1$ eigenvalues $\lambda_{\alpha}$ in $I_{n}$. Therefore, using that the components of any eigenvector are identically distributed, we have

$$
\begin{aligned}
& \mathbb{P}\left(\exists \mathbf{v} \text { with } H \mathbf{v}=\mu \mathbf{v},\|\mathbf{v}\|=1, \mu \in[-2+\kappa, 2-\kappa] \text { and }\|\mathbf{v}\|_{\infty} \geq \frac{C(\log N)^{9 / 2}}{N^{1 / 2}}\right) \\
& \quad \leq N n_{0} \sup _{n} \mathbb{P}\left(\exists \mathbf{v} \text { with } H \mathbf{v}=\mu \mathbf{v},\|\mathbf{v}\|=1, \mu \in I_{n} \text { and }\left|v_{1}\right|^{2} \geq \frac{C(\log N)^{9}}{N}\right) \\
& \quad \leq \operatorname{const} N^{2} \sup _{n} \mathbb{P}\left(\sum_{\lambda_{\alpha} \in I_{n}} \xi_{\alpha} \leq \frac{4 N \eta^{*}}{C}\right) \\
& \quad \leq \operatorname{const} N^{2} \sup _{n} \mathbb{P}\left(\sum_{\lambda_{\alpha} \in I_{n}} \xi_{\alpha} \leq \frac{4 N \eta^{*}}{C} \text { and } \mathcal{N}_{I_{n}} \geq \varepsilon N \eta^{*}\right)+\operatorname{const} N^{2} \sup _{n} \mathbb{P}\left(\mathcal{N}_{I_{n}} \leq \varepsilon N \eta^{*}\right) \\
& \quad \leq \operatorname{const} N^{2} e^{-c(\log N)^{9}}+\operatorname{const} N^{2} e^{-c(\log N)^{2}} \leq e^{-c^{\prime}(\log N)^{2}},
\end{aligned}
$$

by choosing $C$ sufficiently large, depending on $\kappa$ via $\varepsilon$. Here we used Corollary 2.4. of [5] that states that under condition $\mathrm{C} 1$ ) in (1.2) there exists a positive $c$ such that for any $\delta$ small enough

$$
\mathbb{P}\left(\sum_{\alpha \in A} \xi_{\alpha} \leq \delta m\right) \leq e^{-c m}
$$

for all $A \subset\{1, \cdots, N-1\}$ with cardinality $|A|=m$. We remark that by applying Lemma 4.1 from the Appendix instead of Lemma 2.3 in [5], the bound (4.5) also holds without condition C1) if the matrix elements are bounded random variables. It is clear that the boundedness assumption in Lemma 4.1 can be relaxed by performing an appropriate cutoff argument; we will not pursue this direction in this article.

\section{Appendix: Removal of the assumption C1)}

Jean Bourgain

School of Mathematics 


\section{Institute for Advanced Study \\ Princeton, NJ 08540, USA}

The following Lemma shows that the assumption C1) in Lemma 2.3 and its corollary in [5] can be removed.

Lemma 4.1 Suppose that $z_{1}, \ldots, z_{N}$ are bounded, complex valued i.i.d. random variables with $\mathbb{E} z_{i}=0$ and $\mathbb{E}\left|z_{i}\right|^{2}=a>0$. Let $P: \mathbb{C}^{N} \rightarrow \mathbb{C}^{N}$ be a rank-m projection, and $\mathbf{z}=\left(z_{1}, \ldots, z_{N}\right)$. Then, if $\delta$ is small enough, there exists $c>0$ such that

$$
\mathbb{P}\left(|P \mathbf{z}|^{2} \leq \delta m\right) \leq e^{-c m} .
$$

Lemma 2.3 in [5] stated that the same conclusion holds under the condition C1), but it required no assumption on the boundedness of the random variables.

Proof. It is enough to prove that

$$
\mathbb{P}\left(\left.|| P \mathbf{z}\right|^{2}-a m \mid>\tau m\right) \leq e^{-c \tau^{2} m}
$$

for all $\tau$ sufficiently small. Introduce the notation $\|X\|_{q}=\left[\mathbb{E}|X|^{q}\right]^{1 / q}$. Since

$$
\mathbb{P}\left(\left.|| P \mathbf{z}\right|^{2}-a m \mid>\tau m\right) \leq \frac{\left\||P \mathbf{z}|^{2}-a m\right\|_{q}^{q}}{(\tau m)^{q}}
$$

the bound (4.6) follows by showing that

$$
\left\||P \mathbf{z}|^{2}-a m\right\|_{q} \leq C \sqrt{q} \sqrt{m} \quad \text { for all } q<m
$$

(and then choosing $q=\alpha \tau^{2} m$ with a small enough $\alpha$ ). To prove (4.8), observe that (with the notation $\mathbf{e}_{i}=(0, \ldots, 0,1,0 \ldots, 0)$ for the standard basis of $\left.\mathbb{C}^{N}\right)$

$$
|P \mathbf{z}|^{2}=\sum_{i=1}^{N}\left|z_{i}\right|^{2}\left|P \mathbf{e}_{i}\right|^{2}+\sum_{i \neq j}^{N} \bar{z}_{i} z_{j} P \mathbf{e}_{i} \cdot P \mathbf{e}_{j}=a m+\sum_{i=1}^{N}\left(\left|z_{i}\right|^{2}-\mathbb{E}\left|z_{i}\right|^{2}\right)\left|P \mathbf{e}_{i}\right|^{2}+\sum_{i \neq j} \bar{z}_{i} z_{j} P \mathbf{e}_{i} \cdot P \mathbf{e}_{j}
$$

and thus

$$
\left\||P \mathbf{z}|^{2}-a m\right\|_{q} \leq\left\|\sum_{i=1}^{N}\left(\left|z_{i}\right|^{2}-\mathbb{E}\left|z_{i}\right|^{2}\right)\left|P \mathbf{e}_{i}\right|^{2}\right\|_{q}+\left\|\sum_{i \neq j}^{N} \bar{z}_{i} z_{j} P \mathbf{e}_{i} \cdot P \mathbf{e}_{j}\right\|_{q} .
$$

To bound the first term, we use that for arbitrary i.i.d. random variables $x_{1}, \ldots, x_{N}$ with $\mathbb{E} x_{j}=0$ and $\mathbb{E} e^{\delta\left|x_{j}\right|^{2}}<\infty$ for some $\delta>0$, we have the bound

$$
\|X\|_{q} \leq C \sqrt{q}\|X\|_{2}
$$

for $X=\sum_{j=1}^{N} a_{j} x_{j}$, for arbitrary $a_{j} \in \mathbb{C}$. The bound (4.11) is an extension of Khintchine's inequality and it can be proven as follows using the representation

$$
\|X\|_{q}^{q}=q \int_{0}^{\infty} \mathrm{d} y y^{q-1} P(|X| \geq y) .
$$


Writing $a_{j}=\left|a_{j}\right| e^{i \theta_{j}}, \theta_{j} \in \mathbb{R}$, and decomposing $e^{i \theta_{j}} x_{j}$ into real and imaginary parts, it is clearly sufficient to prove (4.11) for the case when $a_{j}, x_{j} \in \mathbb{R}$ are real and $x_{j}$ 's are independent with $\mathbb{E} x_{j}=0$ and $\mathbb{E} e^{\delta\left|x_{j}\right|^{2}}<\infty$. To bound the probability $\mathbb{P}(|X| \geq y)$ we observe that

$$
\mathbb{P}(X \geq y) \leq e^{-t y} \mathbb{E} e^{t X}=e^{-t y} \prod_{j=1}^{N} \mathbb{E} e^{t a_{j} x_{j}} \leq e^{-t y} e^{C t^{2} \sum_{j=1}^{N} a_{j}^{2}}
$$

because $\mathbb{E} e^{\tau x} \leq e^{C \tau^{2}}$ from the moment assumptions on $x_{j}$ with a sufficiently large $C$ depending on $\delta$. Repeating this argument for $-X$, we find

$$
\mathbb{P}(|X| \geq y) \leq 2 e^{-t y} e^{C t^{2} \sum_{j=1}^{N} a_{j}^{2}} \leq e^{-y^{2} /\left(2 C \sum_{j=1}^{N} a_{j}^{2}\right)}
$$

after optimizing in $t$. The estimate (4.11) follows then by plugging the last bound into (4.12) and computing the integral.

Applying (4.11) with $x_{i}=\left|z_{i}\right|^{2}-\mathbb{E}\left|z_{i}\right|^{2}\left(\mathbb{E} e^{\delta x_{i}^{2}}<\infty\right.$ follows from the assumption $\left.\left\|z_{i}\right\|_{\infty}<\infty\right)$, the first term on the r.h.s. of (4.10) can be controlled by

$$
\left\|\sum_{i=1}^{N}\left(\left|z_{i}\right|^{2}-\mathbb{E}\left|z_{i}\right|^{2}\right)\left|P \mathbf{e}_{i}\right|^{2}\right\|_{q} \leq C \sqrt{q}\left(\sum_{i=1}^{N}\left|P \mathbf{e}_{i}\right|^{4}\right)^{1 / 2} \leq C \sqrt{q}\left(\sum_{i=1}^{N}\left|P \mathbf{e}_{i}\right|^{2}\right)^{1 / 2}=C \sqrt{q} \sqrt{m} .
$$

As for the second term on the r.h.s. of (4.10), we define the functions $\xi_{j}(s), s \in[0,1], j=1, \ldots, N$ by

$$
\xi_{j}(s)=\left\{\begin{array}{ll}
1 & \text { if } s \in \bigcup_{k=0}^{2^{j-1}-1}\left[\frac{2 k}{2^{j}}, \frac{2 k+1}{2^{j}}\right) \\
0 & \text { otherwise }
\end{array} .\right.
$$

Since

$$
\int_{0}^{1} \mathrm{~d} s \xi_{i}(s)\left(1-\xi_{j}(s)\right)=\frac{1}{4}
$$

for all $i \neq j$, the second term on the r.h.s. of (4.10) can be estimated by

$$
\left\|\sum_{i \neq j}^{N} \bar{z}_{i} z_{j} P \mathbf{e}_{i} \cdot P \mathbf{e}_{j}\right\|_{q} \leq 4 \int_{0}^{1} \mathrm{~d} s\left\|\sum_{i \neq j}^{N} \xi_{i}(s)\left(1-\xi_{j}(s)\right) \bar{z}_{i} z_{j} P \mathbf{e}_{i} \cdot P \mathbf{e}_{j}\right\|_{q} .
$$

For fixed $s \in[0,1]$, set

$$
I(s)=\left\{1 \leq i \leq N: \xi_{i}(s)=1\right\} \quad \text { and } \quad J(s)=\{1, \ldots, N\} \backslash I(s) .
$$

Then

$$
\left\|\sum_{i \neq j}^{N} \xi_{i}(s)\left(1-\xi_{j}(s)\right) \bar{z}_{i} z_{j} P \mathbf{e}_{i} \cdot P \mathbf{e}_{j}\right\|_{q}=\left\|\sum_{i \in I(s), j \in J(s)} \bar{z}_{i} z_{j} P \mathbf{e}_{i} \cdot P \mathbf{e}_{j}\right\|_{q}=\left\|\sum_{j \in J(s)} z_{j}\left(\sum_{i \in I(s)} z_{i} P \mathbf{e}_{i}\right) \cdot \mathbf{e}_{j}\right\|_{q} .
$$


Since by definition $I \cap J=\emptyset$, the variable $\left\{z_{i}\right\}_{i \in I}$ and the variable $\left\{z_{j}\right\}_{j \in J}$ are independent. Therefore, we can apply Khintchine's inequality (4.11) in the variables $\left\{z_{j}\right\}_{j \in J}$ (separating the real and imaginary parts) to conclude that

$$
\begin{aligned}
\left\|\sum_{i \neq j}^{N} \xi_{i}(s)\left(1-\xi_{j}(s)\right) \bar{z}_{i} z_{j} P \mathbf{e}_{i} \cdot P \mathbf{e}_{j}\right\|_{q} & \leq C \sqrt{q}\left\|\left(\sum_{j \in J(s)}\left|\left(\sum_{i \in I(s)} z_{i} P \mathbf{e}_{i}\right) \cdot \mathbf{e}_{j}\right|^{2}\right)^{1 / 2}\right\|_{q} \\
& \leq C \sqrt{q}\left\|\sum_{i \in I(s)} z_{i} P \mathbf{e}_{i}\right\|_{q} \leq C \sqrt{q}\|P \mathbf{z}\|_{q}
\end{aligned}
$$

for every $s \in[0,1]$. It follows from (4.14) that

$$
\left\|\sum_{i \neq j}^{N} \bar{z}_{i} z_{j} P \mathbf{e}_{i} \cdot P \mathbf{e}_{j}\right\|_{q} \leq C \sqrt{q}\|P \mathbf{z}\|_{q} .
$$

Inserting the last equation and (4.13) into the r.h.s. of (4.10), it follows that

$$
\left\||P \mathbf{z}|^{2}-a m\right\|_{q} \leq C \sqrt{q}\left(\sqrt{m}+\|P \mathbf{z}\|_{q}\right) .
$$

Since clearly

$$
\|P \mathbf{z}\|_{q} \leq\left\||P \mathbf{z}|^{2}-a m\right\|_{q}^{1 / 2}+\sqrt{a m}
$$

the bound (4.8) follows immediately.

Acknowledgments: We thank the referee for very useful comments on earlier versions of this paper. We also thank J. Bourgain for the kind permission to include his result in the appendix.

\section{References}

[1] Anderson, G. W., Guionnet, A., Zeitouni, O.: Lecture notes on random matrices. Book in preparation.

[2] Bai, Z. D., Miao, B., Tsay, J.: Convergence rates of the spectral distributions of large Wigner matrices. Int. Math. J. 1 (2002), no. 1, 65-90.

[3] Bobkov, S. G., Götze, F.: Exponential integrability and transportation cost related to logarithmic Sobolev inequalities. J. Funct. Anal. 163 (1999), no. 1, 1-28.

[4] Deift, P.: Orthogonal polynomials and random matrices: a Riemann-Hilbert approach. Courant Lecture Notes in Mathematics 3, American Mathematical Society, Providence, RI, 1999.

[5] Erdős, L., Schlein, B., Yau, H.-T.: Semicircle law on short scales and delocalization of eigenvectors for Wigner random matrices. 2007, preprint. arXiv.org:0711.1730.

[6] Guionnet, A., Zeitouni, O.: Concentration of the spectral measure for large matrices. Electronic Comm. in Probability 5 (2000), Paper 14. 
[7] Johansson, K.: Universality of the local spacing distribution in certain ensembles of Hermitian Wigner matrices. Comm. Math. Phys. 215 (2001), no.3. 683-705.

[8] Khorunzhy, A.: On smoothed density of states for Wigner random matrices. Random Oper. Stoch. Eq. 5 (1997), no.2., 147-162.

[9] Ledoux, M.: The concentration of measure phenomenon. Mathematical Surveys and Monographs, 89 American Mathematical Society, Providence, RI, 2001.

[10] Quastel, J, Yau, H.-T.: Lattice gases, large deviations, and the incompressible Navier-Stokes equations, Ann. Math, 148, 51-108, 1998. 